\title{
Agent Based Modelling and Simulation of Pedestrian Crowds in Panic Situations
}

\author{
Mohammed M. Alrashed \& Jeff S. Shamma \\ King Abdullah University of Science and Technology (KAUST) \\ Computer, Electrical and Mathematical Science and Engineering Division (CEMSE) \\ Thuwal, Saudi Arabia 23955-6900 \\ mohammed.alrashed@kaust.edu.sa jeff.shamma@kaust.edu.sa
}

\begin{abstract}
The increasing occurrence of panic stampedes during mass events has motivated studying the impact of panic on crowd dynamics. Understanding the collective behaviors of panic stampedes is essential to reducing the risk of deadly crowd disasters. In this work, we use an agent-based formulation to model the collective human behavior in such crowd dynamics. We investigate the impact of panic behavior on crowd dynamics, as a specific form of collective behavior, by introducing a contagious panic parameter. The proposed model describes the intensity and spread of panic through the crowd. The corresponding panic parameter impacts each individual to represent a different variety of behaviors that can be associated with panic situations such as escaping danger, clustering, and pushing. Simulation results show contagious panic and pushing behavior, resulting in a more realistic crowd dynamics model.
\end{abstract}

Keywords: Pedestrian crowd simulation; Agent based models; Multiagent systems.

\section{Introduction}

The rising frequency of mass events has led to an increase in the risk of crowd stampedes. Despite the tremendous effort of authorities, critical situations keep recurring. The lack of understanding of panic within stampedes still causes hundreds of fatalities each year, not to mention the scarce methodical studies of crowd panic behavior. In crowd stampedes, panic is an essential factor that influences crowd behavior. Panic contagion is a type of herding behavior, where panicking individuals tend to cause social contagion and lead to jamming and life-threatening overcrowding [4, 5]. Moreover, biological studies indicate the role of panic in the change of human reactions, decision-making, and behaviors [2].

Motivated by these circumstances, we develop an agent-based model that uses a panic parameter that can be contagious among pedestrians to influence their behaviors. Furthermore, we introduce aggressive pushing behaviors that cause body collisions and stampedes. The model can simulate the motion of pedestrians and reproduce observed features of crowd dynamics such as lane formation, oscillations at bottlenecks, dynamics at intersections, and transition to uncoordinated movement due to clogging. Moreover, the model can include panic contagion among pedestrians, where the value of the panic parameter of one agent is impacted by the panic parameter value of other nearby agents in a dynamic manner. A direction for future work is the degree to which this model represents actual panic in a crowd through verification and validation to real data of crowd panic behavior.

\section{Methods}

Pedestrian crowd dynamics are generally predictable in high-density crowds where pedestrians cannot move freely. Thus pedestrian crowd dynamics give rise to self-propelling interactions between pedestrians. Although every pedestrian has personal preferences, the motion dynamics can be modelled as a social force in such crowds [1]. These forces are representations of internal preferences and objectives to perform certain actions or movements. 
The proposed model will be presented as an extension of the work in [1]. It uses a blend of physical forces and socio-psychological factors to simulate crowd dynamics. Our goal is to introduce contagious panicking and pushing behavior by introducing proportional-integral dynamics, based on control theory, and a panic parameter with contagion.

In our model, every agent has $P_{i}$ as a panic parameter, whose value ranges from zero to one. The initial value for panic parameter is always zero. However, it increases because of external stimuli in the surrounding environment. The value of the panic parameter is also impacted by the neighboring agent. The spread of panic is modelled as follows:

$P_{i}^{+}=c_{i}\left(z_{i} P_{i}+\sum_{j \in r_{s i}} P_{j} w_{j}\right)+\left(1-c_{i}\right) Q_{i}$

where $c_{i}$ is a constant that represents relative weight of the environment effects versus social influence. Similarly, $w_{j}$ is a constant used to weigh the panic influence caused by the agents in the neighboring area of agent $i$, defined by a circle centered at the position of the agent with radius $r_{s i}$. The constant $z_{i}$ represents personal confidence in an agent's own judgment of the situation. In other words, $1-$ $z_{i}$ represents the amount of influence by the other agents in the vicinity of agent $i$, where $\sum_{j \in r_{s i}} w_{j}+z_{i}=$ 1 , and $Q_{i}$ is a variable representing external stimuli in the surrounding environment such as fire or smoke, life-threatening overcrowding, power outage in a crowded public site.

Our proposed model builds on the microsimulation Helbing model from [1]. To introduce aggressive pushing behaviors to the Helbing model, we added auxiliary states in order to overcome repulsive interaction forces. We propose the following acceleration equation:

$\frac{d v_{i}}{d t}=u_{i}+\frac{1}{m_{i}}\left(\sum_{j(\neq i)} f_{i j}+\sum_{w} f_{i w}\right)$

$u_{i}=k_{P i} e_{i}+f\left(P_{i}\right) \cdot k_{I i} \int_{0}^{t} e_{i} d t$

where repulsive interaction forces $f_{i j}$, $f_{i w}$ keep a separating distance from other pedestrian's, $j$, and walls , $w ; e_{i}=v_{i}^{0}-v_{i}$ represents the error between the desired velocity, $v_{i}^{0}$, and the actual velocity, $v_{i}$; and $m_{i}$ is mass. The function $f\left(P_{i}\right)$ is an increasing function of the panic parameter and weighs the effect of the cumulative integrator action. The increase of $f\left(P_{i}\right)$ leads to the increase in following the desired velocity and hence the behavior to push and to cross the crowd. The constants $k_{P i}, k_{I i}$ are agent proportional-integral controller parameters. It is also possible to have parameters such as maximum and nominal velocity depend on panic as well [3]. The proportional and integrator constants reflect the strength of the agent. In other words, they represent how fast an agent change its states: the bigger the constant, the faster the change. However, the interaction forces will be kept unchanged as in the Helbing model.

\section{Results and Discussion}

The introduction of contagious panic has a significant impact on crowd dynamics. A simulation of a fire evacuation of 400 agents in two connecting rooms was conducted in order to explore the effect of panic in the crowd dynamics as a specific form of collective behavior (see Figure 1). We used the fire and spreading smoke in the left room as the environmental source of panic $Q_{i}$. The simulation result shows panic spreading over to the crowd in the right room causing transition to uncoordinated movement due to pushing behavior at the exit. The stampede at the exit causes significant delay in the evacuation time and leads to a greater number of casualties. Video of the simulation is available at [6]. 
The simulation results shows panicking agents tend to push other agents to increase their chance of survival or to open ways among the crowd. To explore the effect of the added auxiliary states and for the sake of comparison, a simulation of 50 agents in a closed room is conducted both for our model and the Helbing model. In the simulation, one agent (we will refer to it as agent-red) tries to push other agents in its way in order to cross the crowd, while the other 49 agents try to maintain their positions. The environmental source of panic parameter $Q_{i}$ is fixed to zero for all agents, as well as $c_{i}$ for all the agents, which means that panic from agent-red is not contagious. For the Helbing model, pushing behavior is described by increasing the desired velocity. Therefore, the maximum desired velocity of 10 $\mathrm{m} / \mathrm{s}$ was given to agent-red. The simulation shows that agent-red was unable to cross the crowd, although the other agents are not pushing. On the other hand, the proposed model was able to exhibit new pushing behaviour by introducing panic-dependent integrator action. Furthermore, for the same simulation agent-red with panic parameter fixed equal to one was able to cross the crowd, although it was given a desired velocity of only $5 \mathrm{~m} / \mathrm{s}$. The simulation results are available as video at [6].

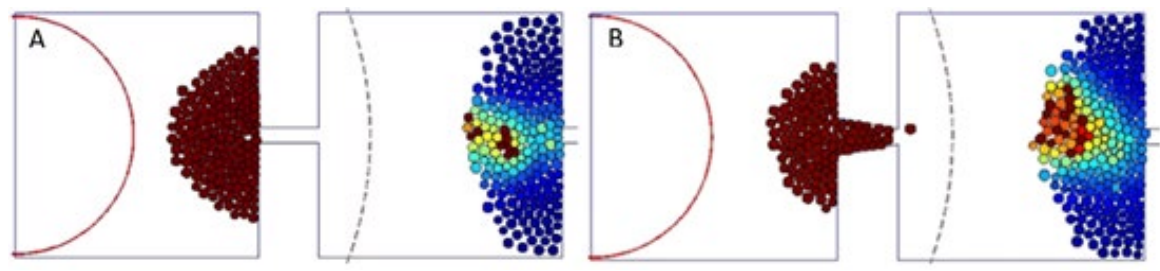

Fig. 1: Panic spreading over to the crowd in the second room.

(A) Zero degrees angle corridor, (B) 10 degrees angle corridor.

We can improve the safety of buildings and facilities by optimizing the boundaries in order to improve the pedestrian flows. Bottleneck boundaries influence pedestrian flows in a crucial way and we can improve the flows by smoothing corners and using funnel shapes. Our objective was to investigate the optimal angle of the funnel shape (see Figure 1). An evacuation simulation of 200 agents with fixed door size and different corridor angles from zero to thirty-five was used to determine an optimal angle. The simulation showed that pressure localization depends on corridor angle. As the angle increases, the pressure moves from the entrance of the corridor to the exit. For the corridor with zero angle, we can see the pressure on an agent in the entrance of the corridor is high. On the other hand, for the corridor with the angle of thirty we can see the pressure on the agent in the exit of the corridor is very high. The results of this simulation show a significant improvement in evacuation time and reduction in the pressure can be achieved by using corridor angle of 10 degrees. The simulation results are available as video at [6].

\section{Acknowledgements}

The research reported in this publication was supported by funding from King Abdullah University of Science and Technology (KAUST).

\section{References}

[1] D. Helbing, I. Farkas, and T. Vicsek, "Simulating dynamical features of escape panic." Nature, vol. 407, no. 6803, pp. 487-490, Sep. 2000.

[2] M.A. Staal, "Stress, Cognition, and Human Performance: A Literature Review and Conceptual Framework", Ames Research Center, Moffett Field, California, NASA/TM-2004-212824.

[3] M. Alrashed, Agent Based Modelling and Simulation of Pedestrian Crowds in Panic Situations, (master dissertation) KAUST. 
[4] Bosse, Tibor, et al. "Agent-based analysis of patterns in crowd behaviour involving contagion of mental states." International Conference on Industrial, Engineering and Other Applications of Applied Intelligent Systems. Springer, Berlin, Heidelberg, 2011.

[5] Elzie, Terra, et al. "Panic that spreads sociobehavioral contagion in pedestrian evacuations." Transportation Research Record: Journal of the Transportation Research Board 2586 (2016): 1-8.

[6] https://www.youtube.com/channel/UCXPNs0in-q2-JFyT3vz_Nnw/videos 